\title{
Pediatric Airway Surgeries in COVID 19 Era
}

\author{
Eshwara V. $\operatorname{Raman}^{1} \cdot$ Deepa Shivnani $^{1}$ (D)
}

Received: 1 July 2020/ Accepted: 20 July 2020/Published online: 27 July 2020

(C) Association of Otolaryngologists of India 2020

\begin{abstract}
To outline a set of recommendations on the management of pediatric cases who requiring airway surgery in the context of COVID 19 pandemic. A set of recommendations have been prepared based on National and International published scientific literature and recent updates on COVID 19. These has been implemented in our tertiary care centre. Due to the evolving nature of COVID 19 and existing knowledge gaps, these recommendations may have to be revised periodically. The incidence of COVID 19 is very low (1-5\%) in the pediatric age group with relatively good prognosis. Pediatric airway surgeries should be restricted to emergency cases only. The decision of postponement of the surgical cases should be taken by the team of senior pediatric airway surgeons. Flexible laryngoscopy should be avoided. Foreign body cases should undergo a computed tomography scan to avoid diagnostic bronchoscopies. All the measures should be taken to prevent direct contact of aerosol so powered instruments should not be used unless mandatory. Protective draping method should be adopted to prevent aerosol exposure. As paediatric airway surgeries are aerosol generating procedure where the risk of contracting COVID 19 by the surgeons and support staff is very high, we suggest recommendations to prevent the contact with infected aerosol. We assure these recommendations are easy to follow and can impact good quality outcome during this pandemic crisis.
\end{abstract}

Deepa Shivnani

deepa.shivnani14@gmail.com

1 Department of Otorhinolaryngology-Head and Neck Surgery, Children's Airway and Swallowing Center, Manipal Hospital, Bangalore, Karnataka, India
Keywords COVID 19 - Pediatric airway surgery · Precautions - Surgical drape

\section{Introduction}

The number of positive or confirmed cases of COVID 19 are much lesser in paediatric age group as compared to adults [1]. Only $2 \%$ of patients were under 19 years of age and $0.9 \%$ under 10 years of age out of 44,672 confirmed cases in a Chinese series [1]. In a Korean series, $4.8 \%$ of patients were under 19 years of age [2]. $1.2 \%$ of patients were younger than 18 out of 22,000 confirmed cases in Italy [3]. And, in the United States, only 5\% are children out of 4000 confirmed cases were found [4]. The prognosis of COVID 19 is good in paediatric population as no death has been reported so far in Italy or China [3, 5-7].

\section{Methods}

Various publications and articles were reviewed to know about current clinical practice guidelines for COVID 19. A set of recommendations has been prepared based on National and International published scientific literature and Recent updates on COVID 19.

These have been implemented in our tertiary care center. Due to the evolving nature of COVID 19 and existing knowledge gaps, these recommendations may have to be revised periodically. 


\section{Results}

These recommendations have been made to help other pediatric airway surgeons to manage airway cases, however, this can be changed during this pandemic crisis though this can be changed later due to the evolving nature of COVID 19 and available evidence. As we know that airway surgeons are at high risk of contracting COVID 19 due to the high concentration of the virus in nasal cavity, nasopharynx and oropharynx. A precise set of recommendations has been documented here which is based on the available evidences and literature [6, 8-12] on COVID 19 at present. There should be a designated team which is thorough with all safety precautions and must be able to perform these procedures with maximum efficiency and safety. Pediatric airway surgeries should be restricted to emergency cases only. The decision of postponement of the surgical cases should be taken by the team of senior pediatric airway surgeons. Flexible laryngoscopy should be avoided. Foreign body cases should undergo CT scan to avoid diagnostic bronchoscopies. All the measures should be taken to prevent direct contact of aerosol so powered instruments should not be used unless mandatory. Protective draping method should be adopted to prevent aerosol exposure.

\section{Discussion}

\section{Precautions to Prevent Infection}

COVID 19 testing should be done prior to all the surgical cases. All cases irrespective of age with unknown COVID status should be considered as positive. Cases should be postponed if it is not urgent or can be manage conservatively. Surgical mask should be provided to all children presenting with symptoms of upper airway infections. During Examination of oral/nasal cavity in OPD: PPE (Personal Protective Equipment-N95 mask, water impermeable gown, disposable gloves, disposable cap, face shield and shoe covers) is highly recommended to prevent any aerosol contact while examining oral or nasal cavity of a child with unknown COVID 19 status.

\section{Planning for Surgical Cases}

Cases should be categorized as follow:

Elective,

Semi elective,

Semi urgent,

Urgent/emergency.
Only urgent or emergency cases should be taken on priority to prevent morbidity or mortality. All children and parents should undergo COVID 19 testing prior to the procedure and should be quarantined till discharged. Designated core COVID Airway team of limited members who can perform Airway procedures efficiently and quickly should be involved. Experienced Senior Surgeons, Anesthetist and senior staff should be involved in the procedure. Complete case discussion should be done among the team prior to patient mobilization into the theatre and before donning to prevent communication problem while using PPE. Precaution should be taken to prevent aerosol generation in all surgical cases. Continuous suction system should be kept near the operative field. Use of electro cautery, powered instrument, drills, coblation device, monopolar tip, microdebrider and laser should be avoided as much as possible. If those are required to be used then PAPR (Powered Air Purifying Respirator) mask should be used. Specific surgical draping (see through plastic drapeFig. 1) should be opted to cover the child in all aerosol generating procedures.

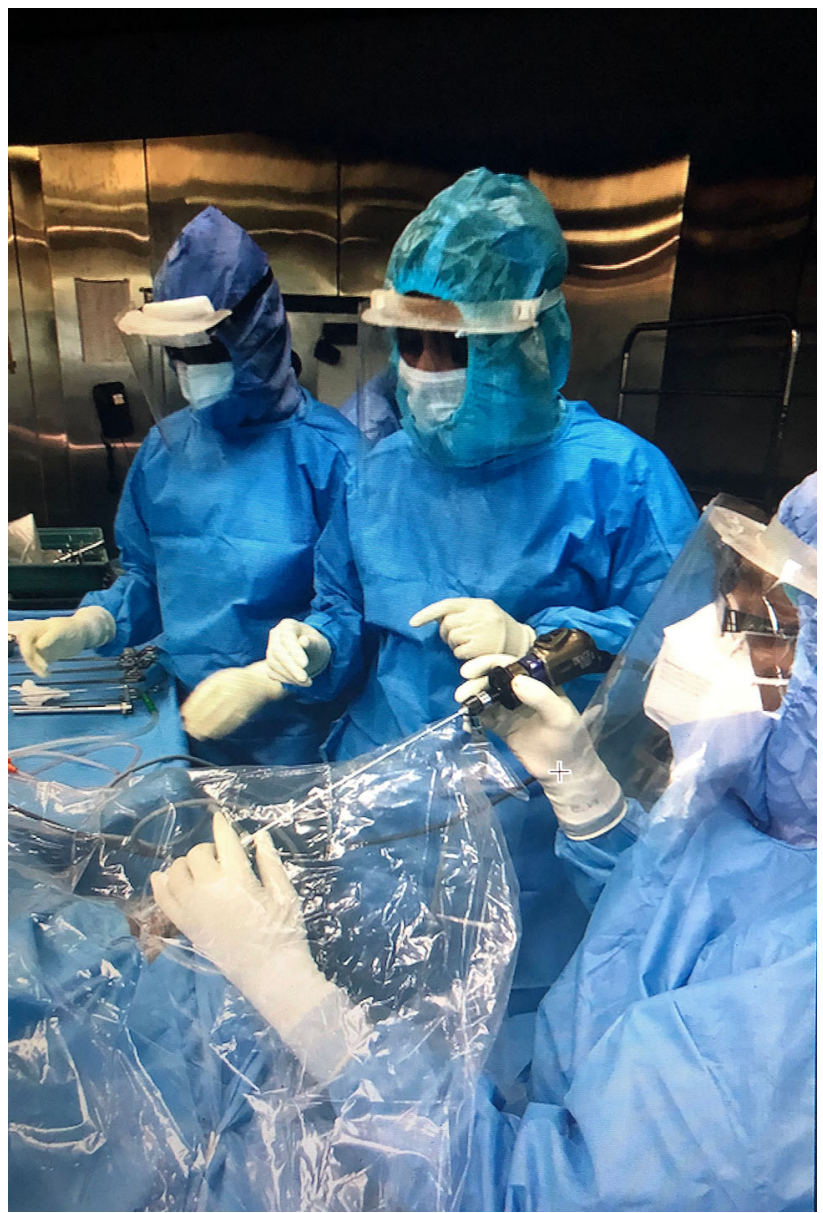

Fig. 1 See-through plastic drape covering the endoscope to prevent aerosol generation while insertion or removal of the instruments 
Extubation should be done under the plastic drape to prevent droplet contamination.

\section{Flexible Laryngoscopy}

Flexible laryngoscopy should be avoided if child is stable. If performed, complete PPE should be worn. Diagnostic flexible laryngoscopy for foreign body should be avoided. A CT scan can be taken for the same to confirm foreign body. Flexible laryngoscopy can be performed with full PPE in case of repeated extubation failures. Nasal cavity should be irrigated with betadine solution (5\% Betadine solution, dilute to $0.5 \%$ in $1: 10$ ratio) prior to the procedure. Care must be taken to sterilize the equipment properly after the use.

\section{Direct Laryngoscopy/Bronchoscopy}

Direct laryngoscopy/bronchoscopy is considered as a highrisk procedure as it is an aerosol generator as well as prolonged open ventilation procedure. Video laryngoscope should be preferred. Sterile transparent/plastic drape/see through protective box with surgeon's arm holes (Fig. 2) should be covered over child's head to prevent aerosol/droplet contact. Instrument handling should be done under the drape to prevent contact with droplets.

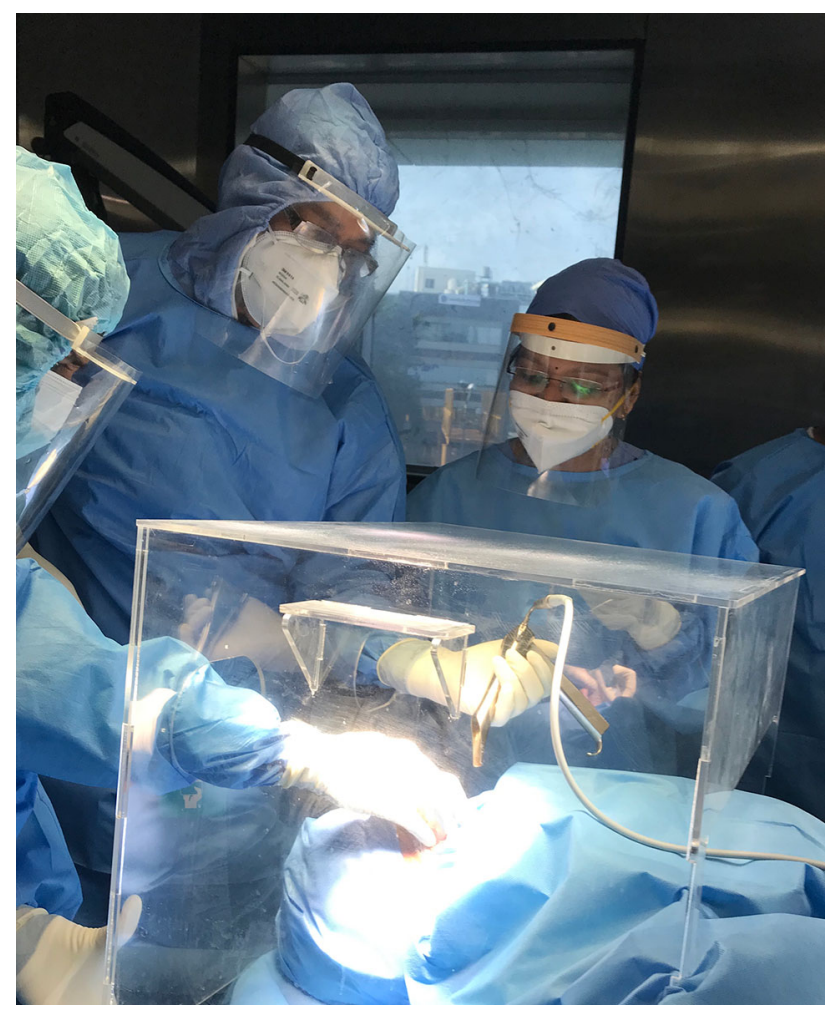

Fig. 2 See-through protective box with the surgeon's arm hole for instrument handling
Continuous close suction should be kept in hypopharynx for continuous suctioning if required. Avoid direct visualization of the surgical field. Direct endoscopic evaluation should be done while performing bronchoscopy. Optical instruments with endoscope and camera should be used in case of foreign body removal. Removal of the instruments from larynx/bronchus should be done slowly to prevent droplets shedding in the surroundings. Care must be taken to keep all the instrumentation under the plastic drape. Drape should be removed with utmost care and with inward folding from all the margins to keep dirty/infected side in.

\section{Tracheostomy}

Preferable site should be OR (Operating Room) rather than ICU (Intensive Care Unit). Elective tracheostomy should be avoided as long as 21 days * post intubation. Once procedure is started the door of the theater should remain closed till the procedure gets over.

* tracheostomy may be performed between 14 and 17 days in high prevalence areas to reduce the burden on ventilators and as early as 10 days in patients with neuromuscular disease.

Only cuffed, non-fenestrated tracheostomy tubes should be used. One circulating sister should arrange heat moisture exchanger (HME) with viral filters beforehand. Patient should be completely paralyzed to reduce risk of coughing. Try to use surgical ties (if needed bipolar) rather than diathermy to prevent virus containing plumes. Every effort should be made not to pierce the cuff of endotracheal tube while giving the tracheal incision. Pause and inform anesthetist of readiness to open trachea. Pre oxygenate with PEEP then stop ventilation and turn off flows before giving incision over trachea. Clearly communicate to cease the ventilation before opening the trachea. Attach syringe to tracheostomy cuff tube for instant inflation of cuff. Immediately inflate tracheostomy tube cuff once tube is in place. Insert inner tube with attached HME with prompt attachment of circuit. Resume ventilation once circuit is connected. Confirm positioning of tube with ET $\mathrm{CO} 2$ (avoid auscultation to prevent contamination of stethoscope). Secure tube with ties and sutures. Use appropriate dressing. Immediately doffing at designated zone of disposal is mandatory.

\section{Tracheostomy Care}

Use only in line closed suction circuits all time. Cuff should not be deflated. Do not change the dressing unless frank signs of infection are noticed. 


\section{Precautions During Tube Change}

Delay first tube change to 7-10 days. Wear full PPE (Personal Protective Equipment). Perform same sequence of pause ventilation before deflating the cuff. Immediately insert the tube, inflate the cuff and connect the circuit. Always use cuffed non-fenestrated tube unless patient is negative for COVID 19. Further tube change can be planned according to the patient's condition. Cuff can be deflated if patient is found negative for COVID 19.

\section{Nasal Foreign Body}

Emergency and critical (button battery) foreign body should be removed to prevent complications. Nose should be decongested with cotton pledgets rather than nasal anesthetic sprays. Endoscopic assisted removal should be done. Foreign body should be removed under the plastic see through cover to prevent droplets contact.

\section{Airway Reconstruction Surgeries}

Open airway surgeries can be avoided if patient is tracheostomized. Endoscopic procedures should be preferred in acute airway compromised cases over tracheostomy or open reconstruction surgeries. COVID 19 testing should be performed prior to open airway procedures. Single stage procedure should be preferred over double stage to avoid tracheostomy and post tracheostomy care for longer duration.

\section{Conclusion}

As paediatric airway surgeries are aerosol generated procedure where the risk of contracting COVID 19 by the surgeons and support staff is very high, we suggest recommendations to prevent the contact with infected aerosol. We assure those recommendations are easy to follow and can impact good quality outcome during this pandemic crisis.

Acknowledgement We sincerely thank Dr.Bathi Reddy for permitting us to take images of operative field while performing surgery.

\section{Compliance with Ethical Standards}

Conflict of interest The authors declare that they have no conflict of interest.
Ethical approval Institutional review board approval was obtained.

\section{References}

1. Novel Coronavirus Pneumonia Emergency Response Epidemiology Team (2020) [The epidemiological characteristics of an outbreak of 2019 novel coronavirus diseases (COVID-19) in China]. Zhonghua Liu Xing Bing Xue Za Zhi 41:145-151. https://doi.org/10.3760/cma.j.issn.0254-6450.2020.02.003

2. Korean Society of Infectious Diseases, Korean Society of Pediatric Infectious Diseases, Korean Society of Epidemiology, Korean Society for Antimicrobial Therapy, Korean Society for Healthcare-associated Infection Control and Prevention, Korea Centers for Disease Control and Prevention (2019) Report on the Epidemiological Features of Coronavirus Disease (COVID-19) Outbreak in the Republic of Korea from January 19 to March 2, 2020. J Korean Med Sci 2020(35):e112. https://doi.org/10.3346/ jkms.2020.35.e112

3. Livingston E, Bucher K (2020) Coronavirus disease 2019 (COVID-19) in Italy. JAMA. https://doi.org/10.1001/jama. 2020.4344

4. Bialek S, Boundy E, Bowen V, Chow N, Cohn A, Dowling N et al (2020) Severe outcomes among patients with coronavirus disease 2019 (COVID-19)—United States, February 12-March 16, 2020. MMWR Morb Mortal Wkly Rep 69:343-346. https://doi.org/10.15585/mmwr.mm6912e2

5. Dong Y, Mo X, Hu Y, Qi X, Jiang F, Jiang Z et al (2019) Epidemiological characteristics of 2143 pediatric patients with 2019 coronavirus disease in China. Pediatrics. https://doi.org/ 10.1542/peds.2020-0702

6. Lu X, Zhang L, Du H, Zhang J, Li YY, Qu J, Chinese Pediatric Novel Coronavirus Study Team et al (2020) SARS-CoV-2 infection in children. N Engl J Med. https://doi.org/10.1056/ NEJMc2005073

7. Wei M, Yuan J, Liu Y, Fu T, Yu X, Zhang Z-J (2020) Novel Coronavirus Infection in Hospitalized Infants Under 1 Year of Age in China. JAMA. https://doi.org/10.1001/jama.2020.2131

8. Brewster DJ, Chrimes NC, Do TB, Fraser K, Groombridge CJ, Higgs A, Humar MJ, Leeuwenburg TJ, McGloughlin S, Newman FG, Nickson CP (2020) Consensus statement: Safe Airway Society principles of airway management and tracheal intubation specific to the COVID-19 adult patient group. Med J Aust 212:472-481

9. Royal College of Anaesthetists UK (2020) COVID-19 airway management principles. https://icmanaesthesiacovid-19.org/ covid-19-airway-management-principles. Accessed 24 Mar 2020

10. ENT UK (2020) Tracheostomy guidance during the COVID-19 pandemic. https://www.entuk.org/tracheostomy-guidance-duringcovid-19-pandemic. Accessed 24 Mar 2020

11. ASOHNS-ASOHNS guidelines addressing the COVID-19 pandemic. 22nd March. http://www.asohns.org.au/

12. WHO 19/3/2020 - Rational use of personal protective equipment (PPE) for coronavirus disease (COVID-19)

Publisher's Note Springer Nature remains neutral with regard to jurisdictional claims in published maps and institutional affiliations. 